\title{
Validation of cardiovascular magnetic resonance assessment of pericardial adipose tissue volume
}

\author{
Adam J Nelson, Matthew I Worthley, Peter J Psaltis, Angelo Carbone, \\ Benjamin K Dundon, Rae F Duncan, Cynthia Piantadosi, Dennis H Lau, \\ Prashanthan Sanders, Gary A Wittert and Stephen G Worthley*
}

Address: Cardiovascular Research Centre, Royal Adelaide Hospital \& Disciplines of Medicine and Physiology, University of Adelaide, Adelaide, SA, Australia

Email: Adam J Nelson - adam.nelson@adelaide.edu.au; Matthew I Worthley - matthew.worthley@adelaide.edu.au;

Peter J Psaltis - peter.psaltis@adelaide.edu.au; Angelo Carbone - angelo.carbone@adelaide.edu.au;

Benjamin K Dundon - benjamin.dundon@adelaide.edu.au; Rae F Duncan - raedavidson7@yahoo.co.uk;

Cynthia Piantadosi - cynthia.piantadosi@adelaide.edu.au; Dennis H Lau - dhslau@gmail.com;

Prashanthan Sanders - prash.sanders@adelaide.edu.au; Gary A Wittert - gary.wittert@adelaide.edu.au;

Stephen G Worthley* - stephen.worthley@adelaide.edu.au

* Corresponding author

Published: 5 May 2009

Journal of Cardiovascular Magnetic Resonance 2009, I I:I5 doi:I0.1 I86/I532-429X-I I-I5

This article is available from: http://www.jcmr-online.com/content/II/I/I5

(C) 2009 Nelson et al; licensee BioMed Central Ltd.

This is an Open Access article distributed under the terms of the Creative Commons Attribution License (http://creativecommons.org/licenses/by/2.0), which permits unrestricted use, distribution, and reproduction in any medium, provided the original work is properly cited.
Received: 15 September 2008

Accepted: 5 May 2009

\begin{abstract}
Background: Pericardial adipose tissue (PAT) has been shown to be an independent predictor of coronary artery disease. To date its assessment has been restricted to the use of surrogate echocardiographic indices such as measurement of epicardial fat thickness over the right ventricular free wall, which have limitations. Cardiovascular magnetic resonance (CMR) offers the potential to noninvasively assess total PAT, however like other imaging modalities, CMR has not yet been validated for this purpose. Thus, we sought to describe a novel technique for assessing total PAT with validation in an ovine model.

Methods: II merino sheep were studied. A standard clinical series of ventricular short axis CMR images (I.5T Siemens Sonata) were obtained during mechanical ventilation breath-holds. Beginning at the mitral annulus, consecutive end-diastolic ventricular images were used to determine the area and volume of epicardial, paracardial and pericardial adipose tissue. In addition adipose thickness was measured at the right ventricular free wall. Following euthanasia, the paracardial adipose tissue was removed from the ventricle and weighed to allow comparison with corresponding CMR measurements.

Results: There was a strong correlation between CMR-derived paracardial adipose tissue volume and ex vivo paracardial mass $\left(R^{2}=0.89, p<0.00 I\right)$. In contrast, $C M R$ measurements of corresponding $R V$ free wall paracardial adipose thickness did not correlate with ex vivo paracardial mass $\left(R^{2}=0.003, p=0.878\right)$.

Conclusion: In this ovine model, CMR-derived paracardial adipose tissue volume, but not the corresponding and conventional measure of paracardial adipose thickness over the RV free wall, accurately reflected paracardial adipose tissue mass. This study validates for the first time, the use of clinically utilised CMR sequences for the accurate and reproducible assessment of pericardial adiposity. Furthermore this non-invasive modality does not use ionising radiation and therefore is ideally suited for future studies of PAT and its role in cardiovascular risk prediction and disease in clinical practice.
\end{abstract}




\section{Background}

Pericardial adipose tissue (PAT) is the layer of fat that surrounds the heart. It covers $80 \%$ of the heart and constitutes between 20 and $50 \%$ of its mass[1]. Historically, this adipose layer has largely been regarded as an inert layer that, at most, may provide some mechanical protection to the coronary arteries[2]. More recently however, the benign nature of this fatty tissue is being re-evaluated.

Pericardial adipose tissue is largely brown adipose tissue[3] and is divided into two layers. The visceral, epicardial fat layer is mainly located within the interventricular and atrioventricular grooves, with lesser amounts located around the atria and right ventricle. This visceral pericardial layer (or epicardial layer) originates embryologically from mesothelial cells that migrate from the septum transversum and hence obtains its vascular supply from the coronary arteries $[4,5]$. The paracardial fat (or mediastinal fat) is situated external to the parietal layer of the pericardium. This layer originates from the primitive thoracic mesenchymal cells and thus derives its blood supply from non-coronary sources such as the pericardiacophrenic branch of the internal mammary artery[5].

Although the pathophysiological significance of PAT remains uncertain, it has recently become the subject of increasing attention, with emerging evidence that it may be an independent predictor of obstructive coronary artery disease [6-8] and possibly acute coronary syndrome[6]. Therefore the characterisation of PAT by noninvasive imaging looms as a potentially valuable adjunct in evaluating cardiovascular risk. There are several candidate modalities for non-invasive imaging of pericardial fat with an ideal methodology providing robust and reproducible data with high accuracy when validated against $e x$ vivo fat mass measurement. Transthoracic echocardiography has traditionally been used to measure epicardial adipose thickness[9], however variable image quality remains a significant limitation particularly in obese subjects: a patient cohort in whom quantification of adipose tissue may be especially relevant. More recently, cardiac computed tomography (CT) $[8,10,11]$ and cardiovascular magnetic resonance (CMR)[12] have been applied with encouraging results. Although magnetic resonance imaging is recognised as the "gold standard" modality for imaging adipose tissue[13,14], CMR has only recently evolved to assess adiposity around the heart[12]. The accuracy of this methodology, however, is yet to be verified against actual measurement of ex vivo mass. In this paper we describe a novel method of assessing complete PAT by volume, and additionally, validate ovine paracardial adipose mass collected at necropsy with its corresponding CMR derived volume. Furthermore we show that volumetric assessment of adipose tissue is a more accurate measure of adipose tissue mass compared with surrogates such as adipose tissue thickness at the RV free wall.

\section{Methods}

An ovine model was chosen to allow direct comparison between CMR-derived measurement of paracardial tissue volume and paracardial tissue mass at necropsy. In addition, a secondary analysis was performed to determine whether single thickness measurement of adipose tissue over the right ventricular (RV) free wall (a parameter routinely used in echocardiographic studies) reflects an accurate surrogate assessment compared to the corresponding adipose tissue mass measured at necropsy.

\section{Cohort}

Approval was obtained from the Animal Ethics Committees of the Institute of Medical and Veterinary Services, Adelaide and the University of Adelaide, South Australia. Animal handling was carried out humanely by dedicated staff in accordance with the "Principles of Laboratory Care" formulated by the National Society for Medical Research and the "Guide for the Care and Use of Laboratory Animals" prepared by the Institute of Laboratory Research and published by the National Institutes of Health.

All animals underwent comprehensive CMR assessment immediately prior to euthanasia at which time heart specimens were harvested for histopathological analysis.

\section{Cardiovascular magnetic resonance protocol}

Cardiovascular magnetic resonance images were acquired using a 1.5 Tesla system (Siemens, Sonata, Erlangen, Germany). Animals were anaesthetised and placed supine within the MR chamber. The animals were mechanically ventilated which allowed breath-hold sequences to be obtained. Pericardial adipose tissue was assessed using sequential ECG-gated steady state free precession short axis cine sequences acquired for assessment of ventricular function (TR/TE $52.05 \mathrm{~ms} / 1.74 \mathrm{~ms}$, flip angle $70^{\circ}$, matrix $256 \times 150$, FOV $380 \mathrm{~mm}$ with slice thickness $6 \mathrm{~mm}$ with no inter-slice gap). To validate a clinically relevant protocol that is already widely used in human studies, this study intentionally did not incorporate adipose-specific sequences and thus did not require additional imaging time.

\section{Image analysis}

Image analysis was carried out off-line using commercially available software, Image Pro Plus (v4 MediaCybernetics). Areas of PAT were traced on consecutive end diastolic short-axis images beginning with the most basal slice at the level of the mitral valve (Figure 1) and moving apically through the stack until the most inferior margin of adipose tissue was traced. The basal slice was taken 


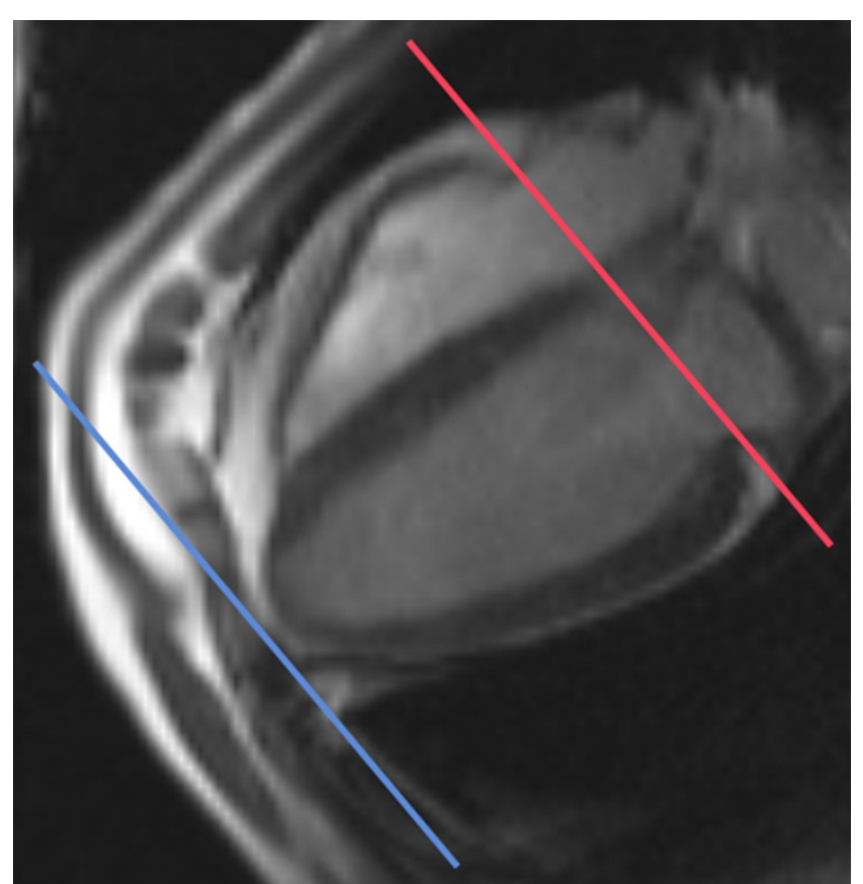

Figure I

Basal and apical slice selection landmarks. Slice through the valve annulus was selected as basal image as this was easiest to approximate for dissection at necropsy.

through the mitral annulus as this was easiest point to dissect at necropsy. Three traces were made on each slice as shown in Figure 2. The first line was drawn along the myoepicardial border. The second line was drawn at the parietal and visceral pericardial border, subtending an area of epicardial fat between this line and the myo-epicardial border. A third line was drawn along the outermost margin of paracardial fat. Epicardial, paracardial and subsequently pericardial fat areas were calculated and volumes were derived using a modified Simpson's rule.

Pericardial adipose thickness at the mid-right ventricular free wall was separately measured as per equivalent echocardiographic methodology used in previous studies evaluating pericardial adiposity $[9,15]$. As with volumes, adipose thickness was sequentially determined from the epicardial, paracardial and finally pericardial zones (Figure 2).

Intra- and interobserver variability was also assessed for the measures of CMR PAT thickness and volumes as described above.

\section{Ex vivo pericardial fat assessment}

The sheep were euthanased following their final CMR examination. The hearts were surgically excised from the thorax with particular care taken to collect all parts of paracardial fat adherent to the mediastinal cavity. As depicted in Figure 3, an incision was made circumferentially along the atrio-ventricular groove through the pericardium. Ventricular adipose tissue, not atrial adipose tissue, was assessed as this has been previously shown to be an independent predictor of coronary artery disease [8] and it is known atrial PAT is only a minor contributor to total myocardial PAT[16]. The pericardium and adherent paracardial adipose tissue was then peeled back from the heart in an apical direction and weighed immediately.

Only the paracardial fat was weighed as we found, like others have before us[17] that it is virtually impossible to accurately dissect the epicardial adipose tissue from the myocardium. Hence correlation between the epicardial component of PAT mass was not able to be undertaken with its respective CMR measurement.

\section{Statistical Analysis}

Continuous variables are represented as means \pm standard deviation or medians with interquartile ranges in brackets for data as appropriate. Statistical analyses were performed with the Statistical Package for the Social Sciences v15 (SPSS Inc, Chicago, Ill). The mass of paracardial adipose tissue at necropsy was compared with the paracardial adipose volume assessed with CMR using simple linear regression analysis. Two sided p-values of less than 0.05 were considered statistically significant. Intra- and interobserver variability was assessed and data analysed with Bland-Altman plots, and coefficients of variation.

\section{Results}

Eleven Merino sheep ( $49 \pm 7 \mathrm{~kg}$ ) were evaluated. CMR imaging was successfully performed in all animals and all images were of acceptable quality for subsequent analysis. CMR imaging acquisition time was approximately 45 minutes per animal. There were no adverse outcomes as a result of the CMR imaging process.

\section{Image analysis}

We were able to trace, and therefore assess, the epicardial, paracardial (and therefore total pericardial adipose tissue) areas in all cases. CMR measurements of adipose tissue thickness and volumes for epicaridal, paracardial and pericardial regions are shown in table 1 . The mean CMR derived PAT thickness was $0.856 \pm 0.16 \mathrm{~cm}$ and the mean CMR derived PAT volume was $103 \pm 26 \mathrm{~cm}^{3}$.

\section{Ex vivo pericardial fat assessment}

In all animals, ex vivo paracardial adipose tissue was able to be excised and weighed as described (Figure 3). Paracardial adipose tissue mass was highly variable across the eleven sheep (Table 1). The mean ex vivo mass of paracardial adipose tissue in this cohort was $139 \pm 49 \mathrm{~g}$. 


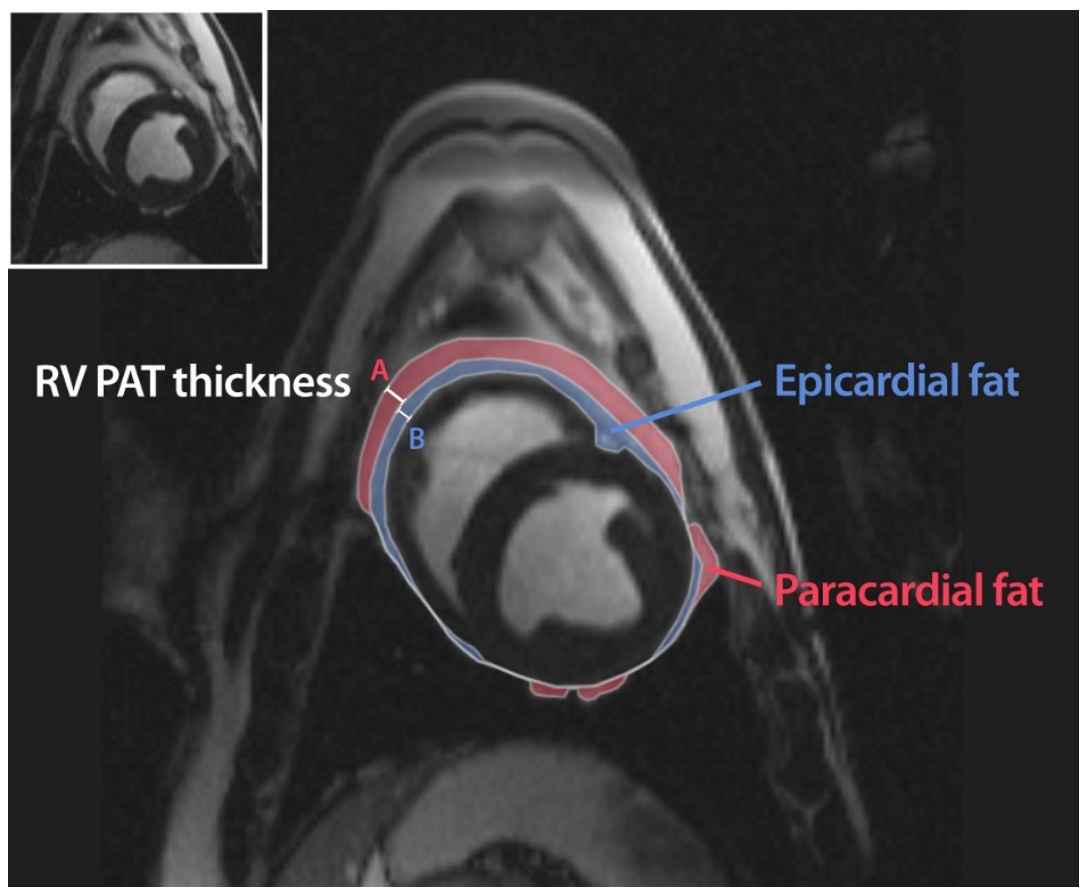

\section{Figure 2}

CMR evaluation of pericardial adipose tissue. Volumetric assessment: 3 traces, myo-epicardial border, viscero-parietal pericardial border and outer paracardial margin each subtending areas of epicardial and paracardial (and subsequently pericardial) areas which can then be converted to volumes. Thickness assessment: adipose tissue thickness measured at the midpoint of the right ventricular free wall on the mid chamber short axis image. Epicardial (A) and paracardial (B) thickness measurements depicted above.

\section{Statistical Analysis}

In regards to the primary hypothesis, there was a strongly positive correlation between CMR derived paracardial adipose tissue volume and ex vivo paracardial adipose mass at necropsy $\left(\mathrm{R}^{2}=0.89, \mathrm{p}<0.001\right)$ (Figure 4$)$. Notably, neither RV free wall paracardial nor pericardial fat thickness correlated with ex vivo PAT mass $\left(\mathrm{R}^{2}=0.003, \mathrm{p}=0.878\right.$ and $\mathrm{R}^{2}=0.33, \mathrm{p}=0.63$ respectively) (Figure 5 ).

Intra and inter-observer coefficients of variation for CMR PAT volumes were $3.5 \%$ and $4.9 \%$ respectively (Table 2 ). The intra and inter-observer coefficients of variation for CMR PAT thickness were higher at 6.7 and $7.6 \%$ respectively (Table 2).

In assessing PAT, the reproducibility of total PAT volume was higher than for the individual epicardial and paracardial measures.

\section{Discussion}

This is the first study to describe an accurate and reproducible CMR technique that is capable of measuring not only total PAT by volume but also its constituents, epicardial and paracardial adipose tissue. In addition, we have shown that CMR assessment of adipose tissue volume dis- plays a strong correlation with its corresponding ex vivo adipose mass, validating its use as a robust measure of cardiac adipose tissue. Further, we have shown that conventional RV free wall adipose thickness measurements do not accurately quantify total PAT. Finally, we have described a method for assessing PAT which intentionally utilises image sequences that would normally be acquired during a standard CMR protocol for myocardial structure and function assessment. The images used were not dedicated adipose-specific sequences as is the case in other studies $[9,12]$ and hence our methodology may be applied to a number of clinically indicated human scans without the need for additional imaging time.

There is growing evidence to support an important role for PAT in the pathophysiology of cardiovascular disease. Although there has recently been a resurgence of interest in this area, the initial descriptions of pericardial adiposity date back hundreds of years. Its initial association with adverse events was described by Senac in 1783 who described "a case of sudden death in which fat spread over the heart extinguishing its movement"[18]. Furthermore the diagnosis of 'adipositas cordis' was a fashionable diagnosis in Victorian times, although the continued association with sudden cardiac death was more than likely 

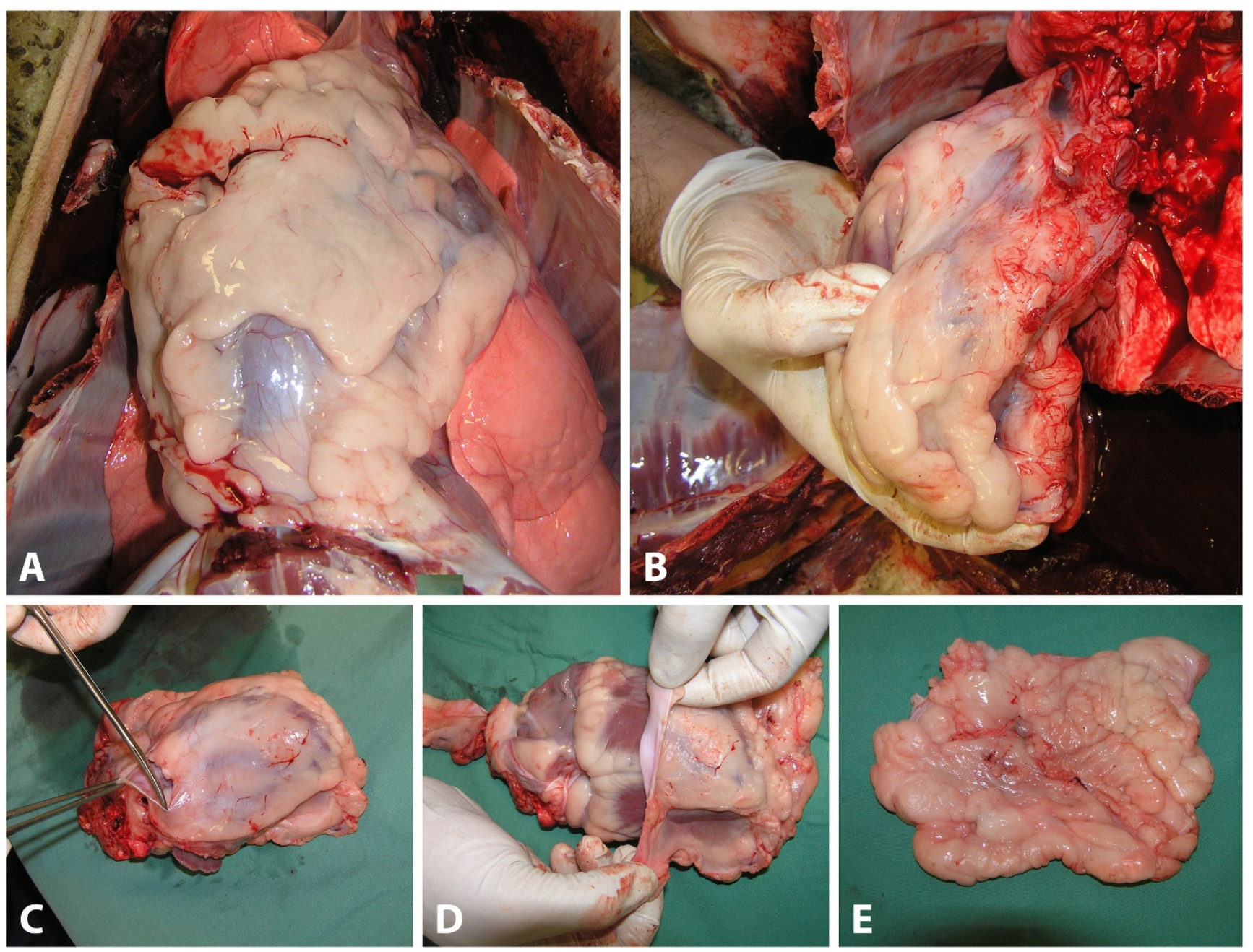

Figure 3

Removal of pericardial fat at necropsy. A) Ovine heart in situ. B) Careful removal of heart collecting adherent fat. C) Cutting through pericardium at the mitral annulus - equivalent point for CMR measurements. D) Removal of PAT from heart by peeling layers back apically. E) Complete removal of PAT before weighing.

related to sudden coronary thrombotic occlusion rather than myocardial fatty infiltration[18]. It was James Herrick's seminal recognition of the clinical syndrome of myocardial infarction in the early $19^{\text {th }}$ century that led to diminished interest in pericardial adiposity.

Much of the initial work performed to quantify epicardial fat was performed at autopsy $[1,17,19,20]$, with the epicardial adipose tissue weighing on average 50 grams and thus representing approximately $20 \%$ of the heart's mass. More recently, echocardiography has been used to evaluate epicardial fat by measuring its thickness over the RV free wall: a prominent site for adipose tissue around the heart $[9,21]$. Indeed epicardial fat thickness has been shown to be a predictor of intra-abdominal visceral fat[9,15], BMI[7,22], left ventricular mass[23] and insulin resistance[24]. Despite this, our ovine study did not find a correlation between CMR measures of RV free wall epicardial or pericardial adipose thickness and the actual mass of pericardial fat at necropsy. We acknowledge however, that there may still be some utility in using epicardial fat thickness in ongoing research of cardiovascular risk assessment, particularly given the accessibility and affordability of transthoracic echocardiograpy compared to CMR. However, the imperfect relationship between this echocardiographic index and PAT, in addition to its lower reproducibility, mean that much larger numbers of patients will be required in such studies. Our CMR technique allows future studies of PAT to show potential alterations or even changes in PAT in response to various therapeutic interventions without the need for prohibitively large sample sizes. 
Table I: Table of CMR and necropsy measurements of PAT. Individual CMR and necropsy measurements of epicardial, paracardial and pericardial adipose tissue in the II sheep studied.

\begin{tabular}{|c|c|c|c|c|c|c|c|}
\hline & \multicolumn{3}{|c|}{ Adipose tissue thickness at mid RV } & \multicolumn{3}{|c|}{ Adipose tissue volume } & \multirow{2}{*}{$\begin{array}{r}\text { Pericardial adipose tissue mass } \\
\text { At necropsy } \\
(\mathrm{g})\end{array}$} \\
\hline & Epicardial (mm) & Paracardial (mm) & Pericardial (mm) & Epicardial $\left(\mathrm{cm}^{3}\right)$ & $\begin{array}{r}\text { Paracardial } \\
\left(\mathrm{cm}^{3}\right)\end{array}$ & Pericardial $\left(\mathrm{cm}^{3}\right)$ & \\
\hline I & 5.21 & 3.52 & 8.73 & 27.493 & 56.957 & 84.45 & 99.22 \\
\hline 2 & 4.62 & 4.28 & 8.9 & 59.37 & 64.56 & 123.93 & 141.68 \\
\hline 3 & 4.45 & 4.74 & 9.19 & 49.194 & 61.122 & 110.316 & 142.4 \\
\hline 4 & 5.63 & 3.81 & 9.44 & 52.836 & 90.852 & 143.688 & 221.2 \\
\hline 5 & 3.39 & 4.84 & 8.23 & 38.21 & 65.614 & 103.824 & 145.3 \\
\hline 6 & 6.08 & 3.91 & 9.99 & 56.521 & 83.723 & 140.244 & 228.3 \\
\hline 7 & 5.28 & 5.16 & 10.44 & 21.476 & 57.436 & 78.912 & 91 \\
\hline 8 & 5.39 & 4.02 & 9.41 & 34.18 & 57.806 & 91.986 & 125.1 \\
\hline 9 & 4.18 & 2.25 & 6.43 & 24.552 & 60.252 & 84.804 & 125 \\
\hline 10 & 0.81 & 4.07 & 4.88 & 30.036 & 78.144 & 108.18 & 139.4 \\
\hline 11 & 4.97 & 3.28 & 8.25 & 19.569 & 40.719 & 60.288 & 72.18 \\
\hline
\end{tabular}

While echocardiography has obvious benefits it also has limitations: most notably its reliance on optimal imaging windows which are notoriously difficult in obese patients[25]. In one study, evaluating over 900 patients from an outpatient echocardiography service, $14 \%$ of obese subjects had poor echocardiographic images[26]. This is relevant given that these high-risk patients may ultimately benefit from cardiovascular risk prediction assessment from non-invasive characterisation of epicardial and/or pericardial fat. By comparison, CMR is heralded for its ability to provide excellent tissue resolution in such patients. Echocardiography is also limited in its ability to measure the complete pericardial adipose layer

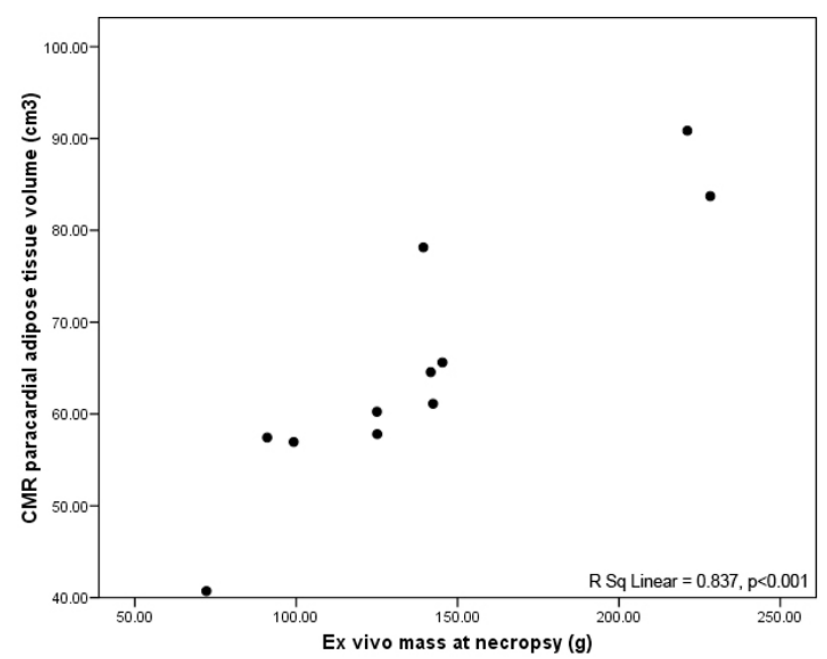

Figure 4

CMR assessed PAT volume versus mass. Linear regression of CMR assessed PAT volume $\left(\mathrm{cm}^{3}\right)$ with ex vivo mass at necropsy $(\mathrm{g}) . \mathrm{R}^{2}=0.892, \mathrm{p}<0.001$. and in particular, to determine total pericardial volume. Volumetric assessment of PAT by CT has been shown to predict the presence of significant coronary artery disease[8] and also to be associated with left ventricular structure and function[10] in subgroups of the Framingham Heart Study. However unlike CMR, cardiac CT has the limitation of exposing patients to ionising radiation, which becomes an increasingly important consideration if patients are subjected to serial imaging.

An intriguing issue that has remained unresolved in the area of pericardial adipose assessment relates to the

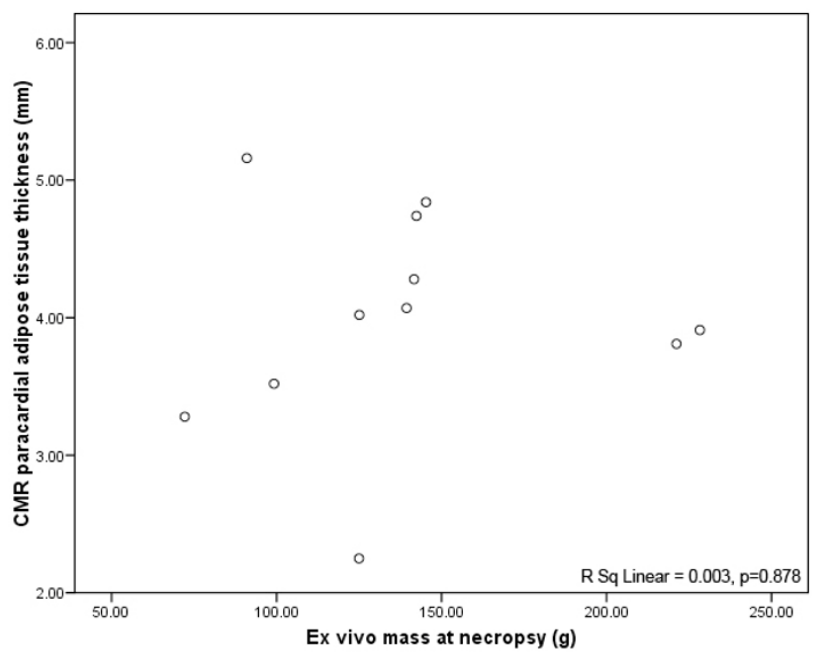

\section{Figure 5}

CMR assessed PAT thickness at right ventricular free wall versus mass. Linear regression of CMR assessed PAT thickness at right ventricular free wall $(\mathrm{cm})$ with ex vivo mass at necropsy $(\mathrm{g})$. $\mathrm{R}^{2}=0.334, \mathrm{p}=0.63$. 
Table 2: Intra and interobserver reproducibility of PAT components.

\begin{tabular}{|c|c|c|c|c|}
\hline & & Mean $(x, y)$ & Mean $(x-y)$ & Coefficient of variation \\
\hline & & & Intra-observer & \\
\hline \multirow{4}{*}{ Thickness } & & {$[\mathrm{cm}]$} & {$[\mathrm{cm}]$} & [\%] \\
\hline & Epicardial & 0.485 & 0.028 & 7.6 \\
\hline & Paracardial & 0.398 & 0.015 & 7.3 \\
\hline & Pericardial & 0.883 & 0.034 & 6.7 \\
\hline \multirow[t]{5}{*}{ Volume } & & {$\left[\mathrm{cm}^{3}\right]$} & {$\left[\mathrm{cm}^{3}\right]$} & [\%] \\
\hline & Epicardial & 38.925 & 0.384 & 4.8 \\
\hline & Paracardial & 61.900 & 0.693 & 4.7 \\
\hline & Pericardial & 103.174 & 1.077 & 3.5 \\
\hline & & & Inter-observer & \\
\hline \multirow[t]{4}{*}{ Thickness } & & {$[\mathrm{cm}]$} & {$[\mathrm{cm}]$} & [\%] \\
\hline & Epicardial & 0.499 & 0.023 & 10.4 \\
\hline & Paracardial & 0.404 & 0.030 & 8.1 \\
\hline & Pericardial & 0.904 & 0.053 & 7.6 \\
\hline \multirow[t]{4}{*}{ Volume } & & {$\left[\mathrm{cm}^{3}\right]$} & {$\left[\mathrm{cm}^{3}\right]$} & [\%] \\
\hline & Epicardial & 40.206 & 0.972 & 6.3 \\
\hline & Paracardial & 63.846 & 1.605 & 5.3 \\
\hline & Pericardial & 100.718 & 2.576 & 4.9 \\
\hline
\end{tabular}

Intra and interobserver reproducibility data for thickness and volume measurements of epicardial, paracardial and pericardial adipose tissue.

importance of differentiating between the epicardial and paracardial layers of adipose. The mechanistic relevance of these two zones of fat appears to be quite distinct. They have different embryological origins and vascular supply $[4,5]$. The epicardial fat layer expresses higher levels of pro-inflammatory mediators than subcutaneous fat in patients with established coronary artery disease suggesting a pathophysiological relationship $[27,28]$. Pericardial adipose tissue may also have a detrimental effect on the coronary vasculature due to mechanical restriction and impairment to diastolic coronary flow[29]. As such, the paracardial layer may have greater importance because it remains the main component of total PAT, as confirmed by the current study. Further studies are required to evaluate the relative clinical importance of the individual adipose layers.

\section{Limitations}

Although it was necessary for this study to be performed in animals to allow for validation of in vivo CMR against ex vivo tissue assessment, we do not have reason to doubt the applicability of our findings to the human clinical scenario. Furthermore, we deliberately did not use predefined, fat-specific imaging sequences in our CMR protocols because it was our intention to utilise the exact images which would otherwise be acquired in a routine clinical investigation. Whilst this CMR protocol did not utilise inter-slice gaps in the short axis stack, unpublished data analysed in other studies[30] suggests it would be unlikely to have a significant effect on our results.

\section{Summary}

A simple CMR volumetric technique using standard clinical steady state free precession imaging is the most accurate and reproducible tool currently available for the assessment of pericardial adipose tissue. Furthermore this non-invasive modality does not use ionising radiation and therefore is ideally suited for future studies of pericardial adipose tissue and its role in cardiovascular risk prediction and disease in clinical practice.

\section{Competing interests}

The authors declare that they have no competing interests.

\section{Authors' contributions}

SW, MW and AN designed the study. CP and RD provided input into CR sequence selection. AC, PP, DL and AN collected necropsy data. AN and AC traced the image contours. SW, MW and AN completed the statistical analysis of the data. SW, MW and AN drafted the article. SW, MW and AN created the figures and images. PP, BD, PS and GW were responsible for critical revision of the article and important intellectual content. All authors read and approved the final manuscript.

\section{Acknowledgements}

Mr Adam Nelson is supported by the Florey Medical Research Foundation, University of Adelaide. Dr Peter Psaltis and Dr Ben Dundon are supported by the National Health and Medical Research Council of Australia, the National Heart Foundation of Australia and the Royal Adelaide Hospital through post-graduate medical research scholarships. Dr Dennis Lau is supported by the Earl Bakken Electrophysiology Scholarship, University of Adelaide, a Kidney Health Australia biomedical research scholarship and 
Postgraduate Research Scholarship from the National Health and Medical Research Council of Australia. Dr Prashanthan Sanders and Dr Stephen Worthley supported by the National Heart Foundation of Australia. This research work is, in part, supported by research grant-in-aid from the National Heart Foundation (G O7A 3086).

\section{References}

I. Shirani J, Berezowski K, Roberts WC: Quantitative measurement of normal and excessive (cor adiposum) subepicardial adipose tissue, its clinical significance, and its effect on electrocardiographic QRS voltage. Am J Cardiol 1995, 76(5):4|4-4|8.

2. Schoenmackers ], Willmen HR: [On lipomatosis cordis, its relations to the efficiency and insufficiency of the right ventricle.]. Arch Kreislaufforsch 1963, 40:251-283.

3. Marchington JM, Mattacks CA, Pond CM: Adipose tissue in the mammalian heart and pericardium: structure, foetal development and biochemical properties. Comp Biochem Physiol B 1989, 94(2):225-232.

4. Ho E, Shimada Y: Formation of the epicardium studied with the scanning electron microscope. Dev Biol 1978, 66(2):579-585.

5. Sacks HS, Fain JN: Human epicardial adipose tissue: a review. Am Heart J 2007, I 53(6):907-917.

6. Ahn SG, Lim HS, Joe DY, Kang SJ, Choi BJ, Choi SY, Yoon MH, Hwang GS, Tahk SJ, Shin JH: Relationship of epicardial adipose tissue by echocardiography to coronary artery disease. Heart 2008, 94(3): 7 .

7. Jeong JW, Jeong MH, Yun KH, Oh SK, Park EM, Kim YK, Rhee SJ, Lee EM, Lee J, Yoo NJ, et al.: Echocardiographic epicardial fat thickness and coronary artery disease. Circ J 2007, 7 I (4):536-539.

8. Rosito GA, Massaro JM, Hoffmann U, Ruberg FL, Mahabadi AA, Vasan RS, O'Donnell C], Fox CS: Pericardial fat, visceral abdominal fat, cardiovascular disease risk factors, and vascular calcification in a community-based sample: the Framingham Heart Study. Circulation 2008, II 7(5):605-6I3.

9. lacobellis G, Assael F, Ribaudo MC, Zappaterreno A, Alessi G, Di Mario U, Leonetti F: Epicardial fat from echocardiography: a new method for visceral adipose tissue prediction. Obes Res 2003, I I(2):304-3I0.

10. Fox CS, Gona P, Hoffmann U, Porter SA, Salton CJ, Massaro JM, Levy D, Larson MG, D'Agostino RB Sr, O'Donnell CJ, et al.: Pericardial fat, intrathoracic fat, and measures of left ventricular structure and function: the Framingham Heart Study. Circulation 2009, I I (1 2): | 586-159|.

II. Gorter PM, van Lindert AS, de Vos AM, Meijs MF, van der Graaf Y, Doevendans PA, Prokop M, Visseren FL: Quantification of epicardial and peri-coronary fat using cardiac computed tomography; reproducibility and relation with obesity and metabolic syndrome in patients suspected of coronary artery disease. Atherosclerosis 2008, 197(2):896-903.

12. Fluchter S, Haghi D, Dinter D, Heberlein W, Kuhl HP, Neff W, Sueselbeck T, Borggrefe M, Papavassiliu T: Volumetric assessment of epicardial adipose tissue with cardiovascular magnetic resonance imaging. Obesity (Silver Spring) 2007, I 5(4):870-878.

13. Gronemeyer SA, Steen RG, Kauffman WM, Reddick WE, Glass JO: Fast adipose tissue (FAT) assessment by MRI. Magn Reson Imaging 2000, I8(7):8|5-8|8.

14. Machann J, Thamer C, Schnoedt B, Haap M, Haring HU, Claussen CD, Stumvoll M, Fritsche A, Schick F: Standardized assessment of whole body adipose tissue topography by MRI. J Magn Reson Imaging 2005, 2 I (4):455-462.

15. lacobellis G, Ribaudo MC, Assael F, Vecci E, Tiberti C, Zappaterreno A, Di Mario U, Leonetti F: Echocardiographic epicardial adipose tissue is related to anthropometric and clinical parameters of metabolic syndrome: a new indicator of cardiovascular risk. J Clin Endocrinol Metab 2003, 88(II):5I63-5I68.

16. lacobellis G, Corradi D, Sharma AM: Epicardial adipose tissue: anatomic, biomolecular and clinical relationships with the heart. Nat Clin Pract Cardiovasc Med 2005, 2( I 0):536-543.

17. Reiner L, Mazzoleni A, Rodriguez FL: Statistical analysis of the epicardial fat weight in human hearts. AMA Arch Pathol 1955, 60(4):369-373.

18. Bedford $\mathrm{E}$ : The story of fatty heart. A disease of Victorian times. Br Heart J 1972, 34(I):23-28.
19. Corradi D, Maestri R, Callegari S, Pastori P, Goldoni M, Luong TV, Bordi C: The ventricular epicardial fat is related to the myocardial mass in normal, ischemic and hypertrophic hearts. Cardiovasc Pathol 2004, I 3(6):3 I 3-3 I6.

20. Sons HU, Hoffmann V: Epicardial fat cell size, fat distribution and fat infiltration of the right and left ventricle of the heart. Anat Anz 1986, I6 I(5):355-373.

21. Malavazos AE, Ermetici F, Coman C, Corsi MM, Morricone L, Ambrosi B: Influence of epicardial adipose tissue and adipocytokine levels on cardiac abnormalities in visceral obesity. Int J Cardiol 2007, I 2 I(I): I32-134.

22. Willens HJ, Byers P, Chirinos JA, Labrador E, Hare JM, de Marchena $E$ : Effects of weight loss after bariatric surgery on epicardial fat measured using echocardiography. Am J Cardiol 2007, 99(9): $1242-1245$

23. lacobellis G, Ribaudo MC, Zappaterreno A, lannucci CV, Leonetti F: Relation between epicardial adipose tissue and left ventricular mass. Am J Cardiol 2004, 94(8): I084- 1087.

24. lacobellis G, Leonetti $F$ : Epicardial adipose tissue and insulin resistance in obese subjects. J Clin Endocrinol Metab 2005, 90(I I):6300-6302.

25. Kessels K, Cramer MJ, Velthuis B: Epicardial adipose tissue imaged by magnetic resonance imaging: an important risk marker of cardiovascular disease. Heart 2006, 92(7):962.

26. Finkelhor RS, Moallem M, Bahler RC: Characteristics and impact of obesity on the outpatient echocardiography laboratory. Am J Cardiol 2006, 97(7): 1082-1084.

27. Baker AR, Silva NF, Quinn DW, Harte AL, Pagano D, Bonser RS, Kumar S, McTernan PG: Human epicardial adipose tissue expresses a pathogenic profile of adipocytokines in patients with cardiovascular disease. Cardiovasc Diabetol 2006, 5: I

28. Mazurek T, Zhang L, Zalewski A, Mannion JD, Diehl JT, Arafat H, Sarov-Blat L, O'Brien S, Keiper EA, Johnson AG, et al.: Human epicardial adipose tissue is a source of inflammatory mediators. Circulation 2003, 108(20):2460-2466.

29. Rokey R, Mulvagh SL, Cheirif J, Mattox KL, Johnston DL: Lipomatous encasement and compression of the heart: antemortem diagnosis by cardiac nuclear magnetic resonance imaging and catheterization. Am Heart J 1989, I I 7(4):952-953.

30. Piantadosi C, Duncan RF, Wittert GA, Worthley MI, Nelson AJ, Marwick T, Worthley SG: Effects of diet-induced weight loss in obese males on cardiac and vascular function. European Heart J 2008, 29(Supp I):87I.

\section{Publish with Bio Med Central and every scientist can read your work free of charge}

"BioMed Central will be the most significant development for disseminating the results of biomedical research in our lifetime. "

Sir Paul Nurse, Cancer Research UK

Your research papers will be:

- available free of charge to the entire biomedical community

- peer reviewed and published immediately upon acceptance

- cited in PubMed and archived on PubMed Central

- yours - you keep the copyright

Submit your manuscript here:

http://www.biomedcentral.com/info/publishing_adv.asp
BioMedcentral 